EPJ Web of Conferences 49, 02002 (2013)

DOI: $10.1051 /$ epjconf/20134902002

(C) Owned by the authors, published by EDP Sciences, 2013

\title{
ALICE detector status and upgrade plans
}

\author{
Ken Oyama ${ }^{1, a}$ for the ALICE Collaboration \\ ${ }^{1}$ Physikalisches Institut der Universität Heidelberg, Heidelberg, Germany
}

\begin{abstract}
ALICE is a general-purpose detector dedicated to heavy-ion physics at the CERN LHC. It is designed to address the physics of strongly interacting matter and the quark-gluon plasma at extreme values of energy density and temperature in nucleus-nucleus collisions. An overview of ALICE experiment, its current status and performance, and the future upgrade plan will be discussed.
\end{abstract}

\section{Introduction}

ALICE (A Large Ion Collider Experiment) is a detector complex conceived for the study of heavy-ion physics at the CERN LHC[1]. It is designed to address the physics of strongly interacting matter and the quark-gluon plasma (QGP) at extreme values of energy density and temperature in high-energy nucleus-nucleus collisions. Within its scope are the particle productions in nucleus-nucleus collisions at energies up to $\sqrt{s_{N N}}=5.5 \mathrm{TeV}$. $p p$ collisions serve as a reference for the study in heavy-ion collisions, while asymmetric collision system, e.g. $p$-A, is studied to investigate the influence of nuclear effects of the initial state such as gluon shadowing and saturation. Studying the QGP properties requires an excellent tracking system in an ultra-high particle multiplicity environment with particle identification down to lowest momenta. In this way ALICE is complementary to the other LHC experiments.

\section{ALICE design}

Figure 1 shows a schematic view of the experimental setup of ALICE[1]. The central barrel detectors are contained in a large solenoid with a magnetic field of $0.5 \mathrm{~T}$ and scintillator paddles for cosmic ray trigger (ACORDE) are fixed on top of the solenoid. In the forward direction sits a muon arm with a charge-separating dipole magnet with a $3 \mathrm{Tm}$ integral field.

The central barrel detectors are used for measurements of hadrons, electrons, and photons produced within \pm 45 degrees with respect to a plane perpendicular to the beam axis, corresponding to pseudo-rapidity coverage of $|\eta|<0.9$. It contains the Inner Tracking System (ITS), the large Time Projection Chamber (TPC), the Transition Radiation Detector (TRD) for electron identification, and the Time-of-Flight (TOF) counters for particle identification (PID), covering full azimuth range. Additionally there are an Electromagnetic Calorimeter (EMCAL), a PHOton Spectrometer (PHOS) with PbWO calorimetry, and a

\footnotetext{
ae-mail: oyama@physi.uni-heidelberg.de
}

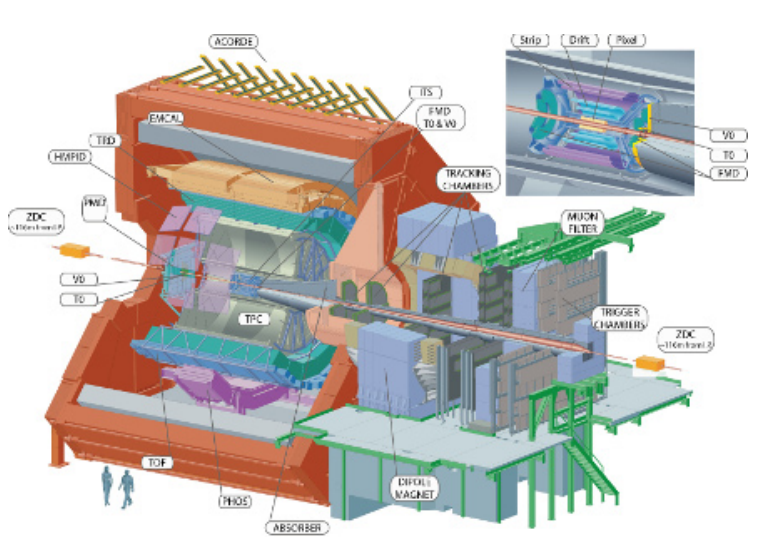

Figure 1. Experimental setup of ALICE.

ring image Čerenkov detector for High-Momentum Particle IDentification (HMPID). These three detectors have limited pseudo-rapidity and azimuthal coverage. The ITS consists of three different silicon detector-technologies, 2 layers of Silicon Pixel Detectors (SPD), 2 layers of Silicon Drift Detectors (SDD), and 2 layers of Silicon Strip Detector (SSD). The arrangement of the detectors is optimized to give good PID performance at below $1 \mathrm{GeV} / c$ using specific energy-loss information and tracking efficiency at expected high multiplicity condition. TRD, EMCAL, and PHOS are essential for triggering for rare probes such as electrons (TRD, EMCAL), photons (EMCAL, PHOS), electrons from decays of heavy quarks (TRD), jets (TRD, EMCAL), and heavy (anti-)nuclei (TRD).

The muon arm detects and identifies muons at pseudorapidity of $2.5<\eta<4$, and has also trigger capabilities for single muons and di-muons to measure heavy quarkonia decay into $\mu^{+} \mu^{-}$. It consists of five pairs of muon tracking chambers, two trigger chambers, an absorber $\left(10 \lambda_{\text {int }}\right)$ and an iron filter wall $\left(7.2 \lambda_{\text {int }}\right)$.

The forward detectors are zero degree calorimeters (ZDC) about $116 \mathrm{~m}$ from the interaction point on each side, a photon multiplicity detector (PMD), a Forward 
Table 1. Summary of data set recorded with ALICE.

\begin{tabular}{crcrr}
\hline system & $\sqrt{s_{N N}}$ & year & min.bias & $\int L d t$ \\
\hline$p p$ & $7 \mathrm{TeV}$ & 2010 & $800 \mathrm{M}$ & $17 \mu \mathrm{b}^{-1}$ \\
$p p$ & $7 \mathrm{TeV}$ & 2011 & $800 \mathrm{M}$ & $2 \mathrm{pb}^{-1}$ \\
$p p$ & $2.76 \mathrm{TeV}$ & 2011 & $70 \mathrm{M}$ & $20 \mathrm{nb}^{-1}$ \\
$p p$ & $8 \mathrm{TeV}$ & 2012 & $500 \mathrm{M}$ & $5 \mathrm{pb}^{-1}$ \\
\hline $\mathrm{Pb}-\mathrm{Pb}$ & $2.76 \mathrm{TeV}$ & 2010 & $30 \mathrm{M}$ & $4 \mu \mathrm{b}^{-1}$ \\
$\mathrm{~Pb}-\mathrm{Pb}$ & $2.76 \mathrm{TeV}$ & 2011 & $9 \mathrm{M}$ & $80 \mu \mathrm{b}^{-1}$ \\
\hline$p-\mathrm{Pb}$ & $5.02 \mathrm{TeV}$ & 2012 & $2.5 \mathrm{M}$ & $0.9 \mu \mathrm{b}^{-1}$ \\
\hline
\end{tabular}

Multiplicity Detector (FMD), and V0 and T0 scintillation detectors. V0 is used as the main interaction trigger, for the measurement of global event properties such as the event plane, and for the measurement of the event centrality in ion collisions. T0 provides a powerful background rejection and determination of the collision timing.

Since ALICE is designed for heavy-ion collisions, it is essential that it can work in an ultra-high multiplicity environment. The anticipated condition for the central barrel is a charged particle density of up to $d N_{c h} / d \eta \sim 8000$. The physics program requires a wide range of $p_{T}$ coverage $(100 \mathrm{MeV} / c$ to $100 \mathrm{GeV} / c)$ with PID to cover identified particle production from the soft to the hard regime. The requirements are achieved by choosing a moderate magnetic field and a low material budget inside the TPC of $X / X_{0} \sim 10 \%$ in range of $0<r<2.5 \mathrm{~m}$ where $r$ is a distance from the interaction point. The choice of the TPC as the central tracking detector gives sufficient particle identification power at a maximum data taking rate of up to $3.5 \mathrm{kHz}$. The upgrade program of ALICE will meet this challenge as discussed in section Sect. 4.3.

\section{ALICE status and performance}

\subsection{Data taking status}

LHC and ALICE have completed the first phase of construction in 2009 after which data taking started. Short runs at intermediate energy were succeeded by longer data taking with $p p$ beams at $\sqrt{s}=7 \mathrm{TeV}$ in 2010-2011. In 2012, LHC increased its energy for $p p$ to $\sqrt{s}=8 \mathrm{TeV}$. Table 1 shows the summary of data sets recorded with ALICE.

The ALICE requirement of an integrated luminosity for proton collisions is $2-5 \mathrm{pb}^{-1}$ per year which is about three orders of magnitude less than ATLAS and CMS. For 2012 data taking, LHC provided the necessary luminosity for ALICE by colliding satellite bunch from one beam with a main bunch from the other beam to reduce luminosity significantly in ALICE and to maximize luminosity in other experiments.

In general, one month per year is reserved for $\mathrm{Pb}-\mathrm{Pb}$ data taking after a 9 months of $p p$ run. At the end of 2010 ALICE successfully recorded the first $\mathrm{Pb}-\mathrm{Pb}$ data. $4 \mu \mathrm{b}^{-1}$ data were collected with a minimum bias trigger setup, corresponding to 30 million $\mathrm{Pb}-\mathrm{Pb}$ collisions. At the end of 2011, LHC increased Pb-Pb luminosity significantly, and in addition to 9 million minimum bias events,
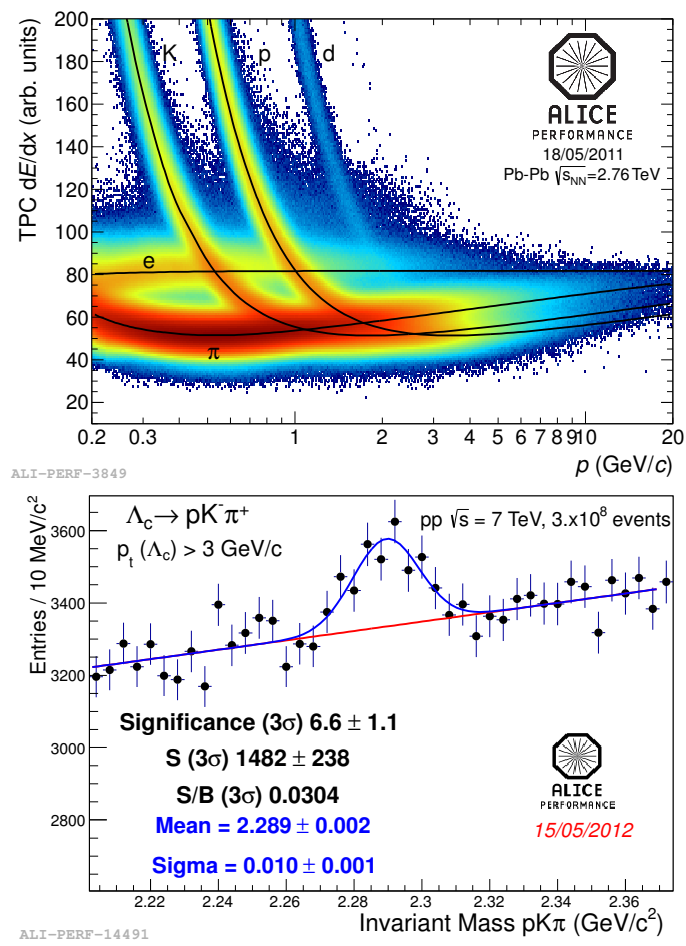

Figure 2. Top: Specific energy loss in the TPC as a function of momentum with superimposed Bethe-Bloch lines for various particle species. Bottom: Invariant mass spectrum of $p \mathrm{~K} \pi$ at $p_{T}>3 \mathrm{GeV} / c$ in $p p$ collisions at $7 \mathrm{TeV}$.

$80 \mu \mathrm{b}^{-1}$ of integrated luminosity were used for rare triggers such as jets and di-muon triggers.

In September 2012, LHC succeeded to collide proton with lead nuclei in a pilot $p$-Pb run, and ALICE has collected $0.9 \mu \mathrm{b}^{-1}$ of data with minimum bias triggers. It is remarkable that the first results for $d N_{c h} / d \eta$ and the nuclear modification factor have been already published[2]. Higher statistics data will be taken in January/February 2013, aiming at $30 \mathrm{nb}^{-1}$.

\subsection{Detector performance}

ALICE has unique tracking and particle identification capabilities down to the lowest momenta. Figure 2 demonstrates the PID and tracking performance of ALICE. As can be seen in the top plot of Figure 2, in the large volume of the TPC, the PID of electrons, pions, and kaons is possible from $200 \mathrm{MeV} / c$, up to $2 \mathrm{GeV} / c$. TRD, TOF and other detectors cover above $2 \mathrm{GeV} / c$.

As shown in Figure 2 bottom plot, the invariant mass spectrum of $p \mathrm{~K} \pi$ was measured in $7 \mathrm{TeV} p p$ collisions, again demonstrating the excellent performance. Reconstructed $\Lambda_{c}$ baryons appear as peak above the combinatorial background at low- $p_{T}$ of $3 \mathrm{GeV} / c$ by powerful PID and tracking performance.

Further physics results of ALICE can be found in the proceedings[2] as well as dedicated material on heavy flavor measurements[3]. 


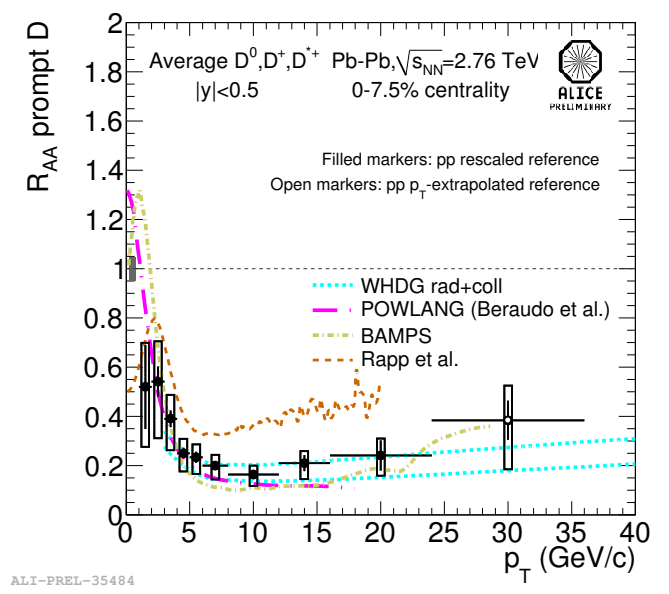

Figure 3. Average $\mathrm{D}^{0}, \mathrm{D}^{+}$and $\mathrm{D}^{*+}$ nuclear modification factor $R_{A A}$ vs $p_{T}$ for central $\mathrm{Pb}-\mathrm{Pb}$ collisions, compared with models.

\section{Upgrade plan of ALICE}

\subsection{Future schedule of LHC and ALICE}

After a successful and long $p p$ operation of LHC in 2012 at $8 \mathrm{TeV}$, LHC will provide, early in 2013, $p$-Pb collisions with an integrated luminosity of $30 \mathrm{nb}^{-1}$ allowing ALICE to disentangle unambiguously initial state effects in $\mathrm{Pb}-\mathrm{Pb}$ collisions.

Directly after the $p$ - $\mathrm{Pb}$ run, LHC will start first long shutdown (LS1) for consolidation of the machine aiming for full design energy of $7 \mathrm{TeV}$ per proton beam. During LS1 ALICE will consolidate sub-detectors such as TRD, PHOS, EMCAL, and SPD.

In 2015-2017, LHC is scheduled for a long run with $p p$ and $\mathrm{Pb}-\mathrm{Pb}$ collisions at full design energy of $\sqrt{s_{N N}}=5.5 \mathrm{TeV}$, followed by the second long shutdown (LS2) starting in 2018. During LS2 LHC will be upgraded to provide 10 times more luminosity and ALICE is planning to undergo major detector upgrades.

\subsection{Physics program after LS2}

To fully understand the property of the QGP, it is fundamental to explore it by means of many different observables, particularly with much higher accuracy. With the upgraded ALICE experiment new opportunities for studies into bulk quark matter arise.

As an example, we will now discuss the predicted improvements in the heavy flavor sector $\left(\mathrm{D}, \mathrm{B}, J / \psi, \psi^{\prime}, \chi_{c}\right.$, $\Lambda_{c}$ ) when going down to $p_{T}=0$. Also, jet production and the response of the medium to propagating jets with identified heavy-quark flavor are of particular interest.

Figure 3 shows the nuclear modification factor $R_{A A}$ for $D$ mesons measured by ALICE in $\mathrm{Pb}-\mathrm{Pb}$ at $\sqrt{s_{N N}}=2.76 \mathrm{TeV}$. The ratio is clearly much less than unity, indicating that heavy quarks suffer substantial energy loss in the hot QGP and thus their production is suppressed when compared to simply binary scaling of $N N$ collisions.

These measurements are indeed intriguing. However, increased statistics and high precision, particularly at low$p_{T}$, are essential to distinguish between various models.
With the upgraded ALICE, differential measurements on heavy quark production, not only D-mesons but also Bmesons, with high precision will be possible.

Further key measurements are the low-mass leptonpair production, hyper (anti-)nuclei, and the search for the postulated H-dibaryon. Measurements of charmed mesons and baryons are particularly important. Enhancement of $J / \psi$ due to recombination of $c \bar{c}$ is predicted for LHC energies since copiously produced charm quarks might thermalize to a high degree[4]. In statistical hadronization models, an increase of $\Lambda_{c}$ production with respect to $\mathrm{D}$ mesons is expected as well, especially at low$p_{T}>2 \mathrm{GeV} / c$.

However measurement of low- $p_{T}$ rare observables such as $\Lambda_{c}$ is quite challenging as it decays into soft hadrons. Presently, no trigger technology exists at the hardware level in such a high multiplicity environment.

\subsection{LS2 upgrade of ALICE}

To meet the physics requirements, ALICE has defined a goal of the statistics to be collected after LS2. This will be about 100 times the data collected before LS2 in order to have reasonable statistical and systematic uncertainties in rare probe measurements. The target is $10 \mathrm{nb}^{-1}$ corresponding to $\sim 10^{10}$ central $\mathrm{Pb}-\mathrm{Pb}$ collisions. To achieve this goal, significant upgrades of both LHC and ALICE are foreseen. LHC will increase the luminosity by a factor of ten, resulting in a $50 \mathrm{kHz} \mathrm{Pb}-\mathrm{Pb}$ interaction rate. The $p p$ reference will be collected at a moderate rate $(\sim 200 \mathrm{kHz})$ which is sufficient since the signal-to-background ratio for most observables is significantly larger.

Given the fact that most of the soft rare probes can not be triggered at the hardware level, it becomes essential to take data with a minimum bias trigger. However the present ALICE setup with the current beam luminosity has the limitation that the trigger live-time is approximately $10 \%$ if ALICE tries to record all minimum bias triggered events. The only solution is to completely remove the trigger dead-time and continuously acquire all collision events with a fully pipe-lined read-out architecture.

This requirement is challenging and ALICE decided for a major upgrade of its sub-detectors (mainly TPC and ITS), the read-out electronics, and the data acquisition system[5, 6].

Additionally, improving the vertexing and tracking capabilities, especially low- $p_{T}$, will further enhance the signal-to-background ratio in the reconstruction of heavyquark decays and might even enable ALICE to fully reconstruct B mesons.

Further upgrade projects are considered to extend the physics scope of ALICE, namely a VHMPID (Very High Momentum Particle IDentification) and a Muon Forward Tracker (MFT). The VHMPID will extend the momentum range for hadron identification up to $p_{T}=25 \mathrm{GeV} / c$. The MFT will add tracking in front of the muon absorber allowing for the reconstruction of secondary vertices from $B \rightarrow J / \psi$ decays as well as low-mass di-muon measurements. Also considered is a forward calorimeter (FOCAL) 

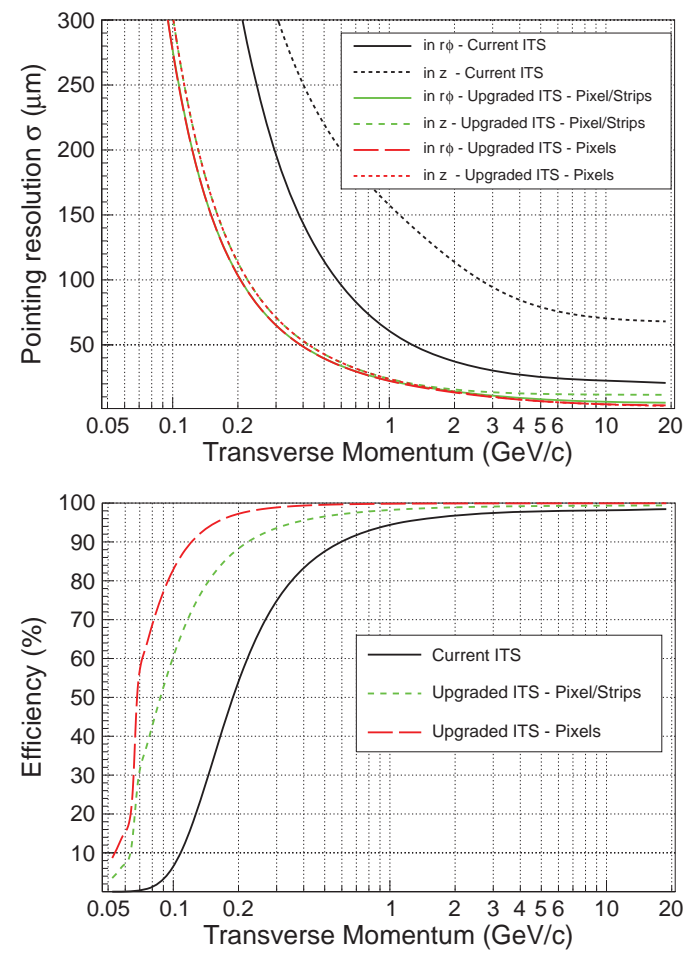

Figure 4. Expected performance of new ITS compared to current ITS. Top: pointing resolution together with TPC as functions of $p_{T}$, for different configurations. Bottom: track finding efficiency with only ITS as functions of $p_{T}$.

to explore low- $x$ physics with identified $\gamma$ and neutral pions. These possible additions are being discussed in the ALICE collaboration.

The high-rate capability and improvement of the tracking capabilities require a full upgrade of several detectors such as the TPC and the ITS, the data acquisition (DAQ) and the high level trigger system (HLT) as well as the front-end electronics of most detectors. The proposed schedule by ALICE is to take data for at least six years with the upgraded detector, until around 2026.

\subsection{ITS upgrade plan}

The redesign of the ITS has been under consideration for several years[6]. The main goal of the upgraded ITS is to significantly improve the pointing (track impact parameter) resolution by a factor of 3 as well as the tracking efficiency. Compared to the current ITS, the upgraded ITS will: (a) be closer to the interaction point which will be achieved by installing a smaller beam pipe $(22 \mathrm{~mm}$ as compared to the present $39 \mathrm{~mm}$ ), (b) have a reduced radiation thickness $\left(X / X_{0}\right)$ per layer going from the present $1.14 \%$ to $0.3 \%$, (c) have a reduced pixel size from the present $50 \times 425 \mu \mathrm{m}^{2}$ to less than $50 \times 50 \mu \mathrm{m}^{2}$, and (d) have an increased number of silicon layers from 6 to 7 .

The layout of the detector is presently under discussion and two different designs are being considered; (a) 7 silicon pixel layers, with $20 \times 20 \mu \mathrm{m}^{2}$ to $50 \times 50 \mu \mathrm{m}^{2}$ pixel size, to have vastly improved tracking efficiency and momentum resolution, or (b) a combination of 4 double sided silicon strip layers (outer barrel) with $95 \mu \mathrm{m} \times 2 \mathrm{~cm}$ and 3 silicon pixel layers (inner barrel) with $20 \times 20 \mu \mathrm{m}^{2}$ pixel size. The latter design is capable of a good $d E / d x$ resolution for PID. Costs of each design and the expected performance are being studied in detail. Monolithic Active Pixel Sensors (MAPS) are some of the candidates under evaluation for the pixel detector technology.

The maximum readout rate of the current SPD is $\sim 3 \mathrm{kHz}$ and that of the SDD is $\sim 1 \mathrm{kHz}$. This readout rate will be improved so that the data is sampled at $40 \mathrm{MHz}$ with continuous full data readout without a trigger.

Figure 4 shows selected plots of the expected performance for the upgraded ITS compared to the current one. The estimate is based on both proposed layouts (a) and (b) described above. For layout (a), a pointing resolution of $4 \times 4 \mu \mathrm{m}^{2}$ was assumed. For layout (b), the same pointing resolution as (a) for the pixel and $20 \times 830 \mu \mathrm{m}^{2}$ for the strip layers was assumed. In each case, the radial positions of each layer were chosen as 22, 28, 36, 200, 220, 410, and $430 \mathrm{~mm}$. We expect that upgraded ITS will have a pointing resolution improved by a factor of 3 in the $r \phi$ and a factor of five in the $z$ direction. This will significantly improve the efficiency for the topological selection of short-lived decay particles, and give about 2 times better $p_{T}$ resolution in the range from $0.1-20 \mathrm{GeV} / c$ for ITS stand-alone tracking. The tracking efficiency is above $90 \%$ at above $600 \mathrm{MeV} / c$ in the current ITS. The same efficiency will be achieved already at $200 \mathrm{MeV} / c$ with the upgraded ITS.

\subsection{TPC upgrade plan}

The present TPC has a long drift time with gating grid operation ultimately limiting the rate capabilities. Using the multi-wire readout chambers, a gated operation is necessary to limit the ion feedback to the drift volume below the $0.1 \%$ level. For this purpose, the gating grid needs to be kept closed for about $180 \mu$ s after it was opened for the maximum drift time of $100 \mu \mathrm{s}$, to collect ions. This limits the maximum readout rate of the TPC to $3.5 \mathrm{kHz}$. The TPC upgrade is a key element in achieving the 100 times increase in recorded data in $\mathrm{Pb}-\mathrm{Pb}$ after LS2.

The high rate operation of the TPC clearly requires to abolish the gating grid operation. However, without gating grid there will be intolerable space-charge distortions at the level of $\sim 1 \mathrm{~m}$ due to a small amount of ion feed-back into the drift volume. In addition, the deterioration in the $d E / d x$ measurement becomes an unacceptably large (10$20 \%$ ) due to decrease of the gain.

To solve the problem of TPC operation without a gating grid, a new readout chambers based on triple-GEM technology is under development[5]. In the proposed design, the pad-plane of the TPC and most of the mechanical structure can be re-used. Using GEMs with continuous readout will result in an acceptably small ion feedback and will allow an operation at $50 \mathrm{kHz} \mathrm{Pb}-\mathrm{Pb}$ interaction rate. However, the use of GEMs in a large TPC has not yet been demonstrated and there are several innovative requirements. $100 \mathrm{~m}^{2}$ of active area has to be covered with large $1 \times 0.8 \mathrm{~m}^{2}$ GEM foils which was recently made possible by single-mask GEM production technology. 


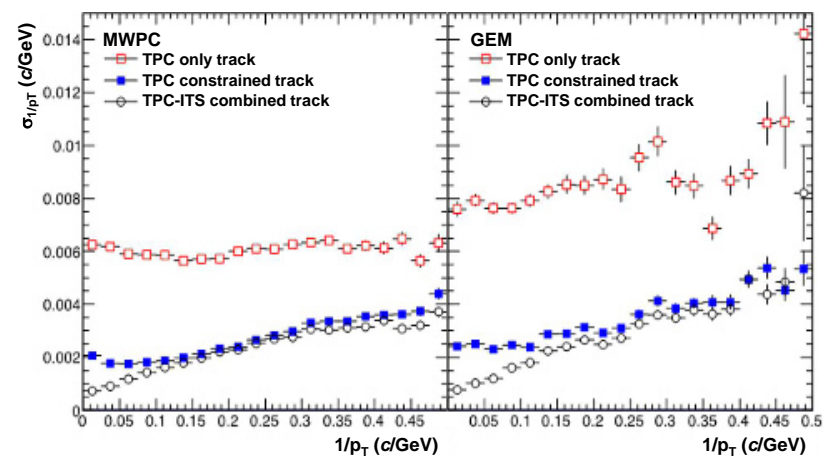

Figure 5. Momentum resolution of the current TPC (left) and the expected resolution of the upgraded TPC (right) as a function of $1 / p_{T}$. Open squares are for TPC information only, closed squares show track fits including the primary vertex, and open circles show the result for combined fits to TPC and ITS tracks.

Figure 5 shows the difference in momentum resolution with the current TPC using MWPC readout and with the upgraded TPC using GEM readout. The GEMs will have, by their characteristics, hardly any spread of signal over several pads since the pads have about $1 \mathrm{~cm}$ size optimized for MWPC readout of the present TPC. Therefore, with a GEM TPC a better spatial resolution than the size of the pads using pad response functions will not be expected if we reuse the pad planes of the present TPC. However, by combining with the upgraded ITS, the overall momentum resolution will be at the same level as for the MWPC-type TPC.

R\&D on the GEM readout chambers is ongoing together with the CERN RD51 group with an emphasis on achieving the projected low ion feedback and in particular on demonstrating the necessary stability in a highmultiplicity and high-rate environment. The ion feed-back fraction has to be below $0.25 \%$ corresponding to a $\sim 5 \mathrm{~cm}$ deterioration in the electron cluster position measurement. Gain stability is essential for stable $d E / d x$ measurements for PID.

\subsection{Online system upgrade}

In a pipe-line readout architecture data will be shipped from the TPC and ITS front-end at $10 \mathrm{MHz}$ (TPC) or $40 \mathrm{MHz}$ (ITS) for later inspection by a high level trigger system (HLT). The readout electronics of all existing detector systems will be upgraded to accommodate for the required rate capabilities.

All detectors with upgraded front-end electronics will share the pipe-lined continuous readout electronics with the TPC and ITS. TOF, which already has a pipelined readout, will improve its readout rate to $200 \mathrm{kHz}$. However other detectors such as EMCAL and TRD, will keep their on-detector electronics unchanged and will be operated with a hardware triggered data acquisition method.

The amount of data shipped from detectors to HLT will be in the order of $1 \mathrm{~TB} / \mathrm{s}$ dominated by data from the TPC. This has to be reduced online in HLT, in particular
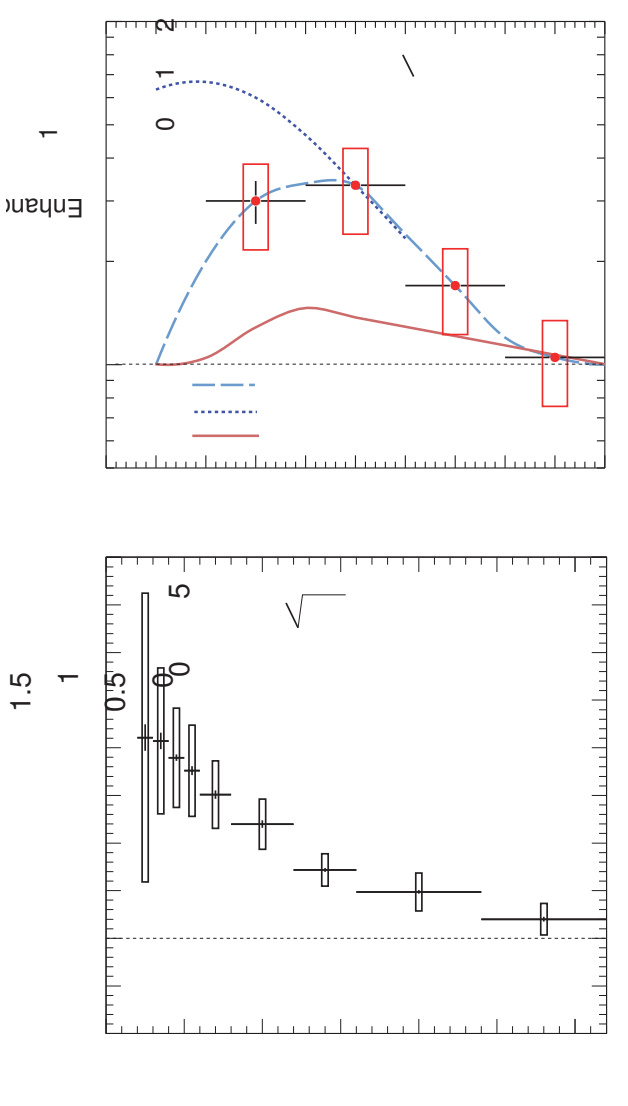

Figure 6. Top: Enhancement of the $\Lambda_{c} / D^{0}$ ratio in central $\mathrm{Pb}$ $\mathrm{Pb}$ collisions with respect to $p p$ collisions, compared with model calculations [7, 8]. Bottom: Expected measurement of the ratio of $\mathrm{D}$ meson yields with feed-down from $\mathrm{B}$ and prompt $\mathrm{D}$, to be measured with the upgraded ALICE.

for the TPC information by cluster finding and cluster-totrack association as well as by standard Huffman encoding. An average output rate to the mass storage system will be $\sim 10 \mathrm{~GB} / \mathrm{s}$ after data compression.

\subsection{Performance of the upgraded ALICE}

The upgrades of ALICE detector and the LHC will open the possibility of many detailed studies in heavy ion collisions. Many aspects of the ALICE upgrade have been studied in preparation for the Letter of Intent $[5,6]$.

The top panel of Figure 6 demonstrates the feasibility of a $\Lambda_{c} / \mathrm{D}$ measurement with upgraded ALICE after LS2, after accumulating data for $10 \mathrm{nb}^{-1}$, and with $1.7 \times 10^{10}$ central events. The high statistics data set collected will allow for a precise measurement of $\Lambda_{c}$ in several $p_{T}$ bins with a factor 10 less statistical errors.

Similar measurements will be feasible also for B hadrons. Quarks propagating in the medium will lose energy due to interactions, e.g. induced gluon radiation. Heavier quarks are predicted to lose less energy, due to a suppression of gluon emission at forward angles (dead cone effect), and are thus less influenced by the medium. However, since there is no precise measurements yet, the quantitative comparison of $c-$ and $b-$ quark energy loss 


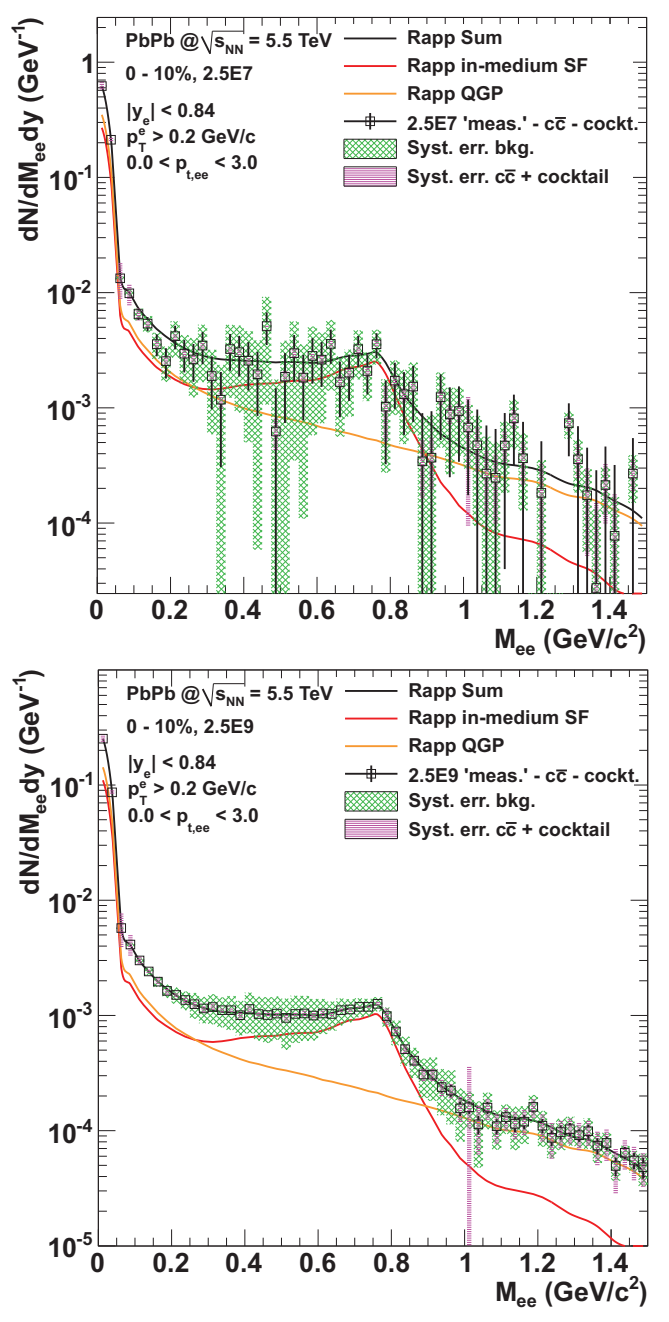

Figure 7. Improved performance of low-mass di-electron measurements without (top) and with (bottom) the upgraded ALICE, after subtracting hadron (cocktail) and charm contributions. The data points have been calculated using a model calculations with hadronic and QGP phase contributions with a realistic space-time evolution, including medium-modified spectral functions [5, 9].

and and heavy-quark hadronization are one of the major goals of ALICE, i.e. determining the quark mass dependence of the energy loss mechanism and possibly heavy quark thermalization. The bottom panel of Figure 6 shows $\mathrm{D}$ mesons $p_{T}$ spectra from the decay of B hadrons. Here, the $\mathrm{B}$ mesons are identified by recognition of their decay vertices, being displaced from the primary vertex, of $\mathrm{D}^{0}$ decaying $\mathrm{K}^{-} \pi^{+}$using tracking with the improved pointing resolution of the upgraded ITS. Without the ITS upgrade the statistical error would be a factor of 10 larger and systematic errors would be greater too.

Measuring low-mass di-electron spectra is of great interest in heavy-ion collisions. A precise in-medium mass spectrum of the $\rho$ meson gives access to the chiral symmetry breaking mechanism. Figure 7 compares the results of low-mass di-electrons with and without the ALICE up- grade. Without the upgrade, ALICE can collect $2.5 \times 10^{7}$ central events whereas we expect 100 times more events with the upgrade. This will result in significantly improved systematic and statistical uncertainties, and allow precise comparison with model calculations. Further, the invariant mass spectra will reach zero-mass by measuring electron momenta down to $0.2 \mathrm{GeV} / c$, allowing for a precise photon measurements from thermal radiation of the medium.

\section{Summary}

ALICE was designed and built as the dedicated apparatus for heavy-ion physics at the LHC. An overview of subdetectors in ALICE and the data taking status have been presented. After having runs with $p p, \mathrm{~Pb}-\mathrm{Pb}$, and $p-\mathrm{Pb}$ successfully, ALICE has entered an exciting stage of new measurements to study strongly interacting partonic matter.

Early in 2013, ALICE plans to collect a significant $p$ $\mathrm{Pb}$ data sample. $\mathrm{LHC}$ and the associated experiments will then have a long shutdown until beginning of 2015. There will then be a long running period with LHC operating at its design energy followed by another long shutdown (LS2) starting in 2018.

During the LS2, ALICE plans to significantly upgrade the detectors and data acquisition system aiming for 100 times higher statistics of about $10 \mathrm{nb}^{-1}$ thus taking full advantage of the upgraded high-luminosity LHC. The upgraded Inner Tracking System with improved pointing resolution, a continuous-readout TPC based on GEM technology, and new online data processing system are key elements of the ALICE upgrade. With the ALICE upgrade, precise study of partonic matter with heavy-quarks covering the full momentum spectrum and low- $p_{T}$ soft observables will be possible.

\section{References}

[1] K. Aamodt et al. (ALICE Collaboration), J. Instrum. 3, S08002 (2008)

[2] R. Preghenella, this proceedings

[3] F. Prino, this proceedings

[4] A. Andronic, P. Braun-Munzinger, and J. Stachel, Phys. Lett. B673, 142 (2009)

[5] The ALICE Collaboration, CERN-LHCC-2012-012 (2012)

[6] The ALICE Collaboration, CERN-LHCC-2012-013 (2012)

[7] S. H. Lee, K. Ohnishi, S. Yasui, I.-K. Yoo, and C. M. Ko, Phys. Rev. Lett. 100, 222301 (2008)

[8] M. He, R. J. Fries, R. Rapp, arXiv:1204.4442 [nucl-th] (2012)

[9] H. van Hees, R. Rapp, arXiv:0711.3444 [hep-ph] (2007) 